\title{
HIGHLIGHTS
}

INTERVENTIONAL CARDIOLOGY

\section{TAVI-transcatheter approach choice does not affect cerebral embolisms}

A new study has found that the transfemoral (TF) and transapical (TA) approaches in transcatheter aortic valve implantation (TAVI) are associated with similar rates of cerebral embolism. Further research into the mechanisms linking TAVI with cerebral embolisms and methods that can be used to prevent them from occurring is, therefore, needed.

Surgical valve replacement is the gold standard in the treatment of patients with symptomatic severe aortic stenosis; however, some patients are unable to undergo an operation or are considered unsuitable for this procedure because they are at very high surgical risk. In the past few years, clinicians have been successfully performing TAVI in such patients at multiple centers around the world.

Excitement about the success of TAVI has been somewhat tempered by the high incidence of cerebral embolisms associated with the intervention. Many have hypothesized that use of a TA approach might result in a lower rate of cerebral embolisms than the TF approach, because the former method avoids the manipulation of large catheters through the ascending aorta and aortic arch and the retrograde crossing of the aorta. Dr Josep Rodés-Cabau and colleagues set out to formally test this hypothesis.

Patients were assigned to TF-TAVI or TA-TAVI on the basis of whether the size and condition of the iliofemoral arteries precluded the use of the TF approach. Cerebral diffusion-weighted (DW)-MRI was performed in all patients in the $24 \mathrm{~h}$ prior to TAVI and then $4 \pm 1$ days after the procedure. Neurological and cognitive function were assessed using the National Institutes of Health Stroke Scale (NIHSS) questionnaire and the Mini Mental State Examination (MMSE), respectively. In total, 29 and 31 patients underwent TAVI using the TF and TA approaches, respectively. Cerebral DW-MRI demonstrated no difference in the number of patients with new lesions after undergoing one of the two approaches -new lesions were detected in $66 \%$ and $71 \%$ of patients who underwent the TF-TAVI and TA-TAVI procedures, respectively. In addition, no differences were observed for the median number of new lesions per patient (3 versus 4) nor the lesion size ( $92 \%$ and $91 \%$ were $<1 \mathrm{~cm}$ in the TF-TAVI and TA-TAVI groups, respectively). The new cerebral embolisms identified by DW-MRI were not associated with any measurable impairment of neurological or cognitive function. One patient from each treatment group had a clinically apparent stroke within the $24 \mathrm{~h}$ period after the intervention.

Given that the choice of transcatheter approach did not affect the high rate of cerebral embolisms associated with TAVI, the investigators postulate that these embolisms might result from the

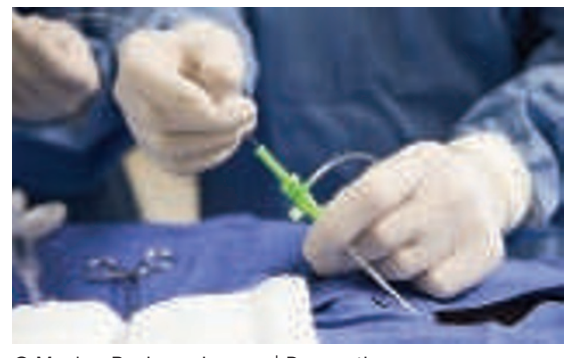

๑) Monkey Business Images | Dreamstime.com

introduction of air though the very large catheters used or, since the aortic valve leaflets are maximally stretched during balloon valvuloplasty and valve implantation, from major mechanical stress during the intervention. Alternatively, the higher embolic rate associated with TAVI, as compared with the surgical method, might simply result from these patients being more sick prior to the procedure.

So, what's the next step? Dr Rodés-Cabau believes that "evaluating the use of embolic protection devices during TAVI procedures might be [an] important [next step] in reducing the rates of cerebral embolism and clinically apparent stroke." In terms of how to best study the efficacy of embolic protection devices in this setting, Dr Rodés-Cabau points out that "the use of silent cerebral embolism as evaluated by MRI as the primary end point ... would allow us to perform such studies with a much smaller sample size."

Bryony M. Mearns

Original article Rodés-Cabau, J. et al. Cerebral embolism following transcatheter aortic valve implantation: comparison of transfemoral and transapical approaches. J. Am. Coll. Cardiol. 57, 18-28 (2011) 